EXTENDED REPORT

\title{
The relation between intraocular pressure peak in the water drinking test and visual field progression in glaucoma
}

\author{
R Susanna Jr, R M Vessani, L Sakata, L C Zacarias, M Hatanaka
}

Br J Ophthalmol 2005;89:1298-1301. doi: 10.1136/bjo.2005.070649

See end of article for authors' affiliations

.....................

Correspondence to: Dr R M Vessani, Rua Itambé 422, apt 52, São Paulo, São Paulo, Brazil; robves@terra.com.br

Accepted for publication 1 April 2005
Aim: To compare the results of the water drinking test between glaucomatous eyes with and without visual field progression.

Methods: Retrospective analysis of 76 eyes of 76 open angle glaucoma patients followed for a mean period of 26.0 (SD 13.8) months. Patients were submitted to the water drinking test at the beginning of the follow up period. Reliable achromatic automated perimetry tests performed during the studied period were used to characterise visual field progression. All subjects were under clinical therapy and had an intraocular pressure (IOP) lower than $17 \mathrm{~mm} \mathrm{Hg}$ monitored by isolated measurements during the follow up period. The results of the water drinking test were compared between glaucomatous eyes with and without visual field progression.

Results: Twenty eight eyes reached definite visual field progression. There were no significant differences in the mean age, sex, race, basal IOP, number of antiglaucomatous drugs, initial mean deviation (MD), and corrected pattern standard deviation (CPSD) between eyes that showed visual field progression and the ones who did not progress. A significant difference of 1.9 (SD 0.6$) \mathrm{mm} \mathrm{Hg} \mathrm{(}=0.001$, analysis of covariance; $95 \% \mathrm{Cl} 0.8$ to 3.0 ) was observed between glaucomatous eyes that showed visual field deterioration and glaucomatous eyes that did not progress. A significant difference of $16.8 \%$ (SD 4.6\%) in the mean percentage of IOP variation was also observed between the two groups $(p<0.001$, analysis of covariance; $95 \% \mathrm{Cl} 7.7$ to 26.0$)$.

Conclusions: Mean IOP peak and percentage of IOP variation during water drinking test were significantly higher in patients with visual field progression compared with patients who did not progress. f ncreased intraocular pressure (IOP) is the main risk factor for the development of glaucoma and its progression. ${ }^{1}$ Lowering IOP is believed to be beneficial in slowing down glaucomatous changes of the optic nerves and visual field. ${ }^{2-4}$ However, reduction of IOP to a preselected level does not always prevent deterioration..$^{5-7}$ Factors independent of IOP may be responsible, in part, for the sustained progression of glaucoma ${ }^{8}$ but it has been suggested that in some cases the progressive damage could be caused by peaks of IOP or diurnal IOP variability not detected by tonometry during office hours. ${ }^{9-12}$

A diurnal IOP curve gives a better estimate of an individual's IOP level and fluctuations than a single measurement during the office visit, but demands hospital admission where IOP is measured over 24 hours. ${ }^{10}$ Some authors have demonstrated "home tonometry" as another form to obtain 24 hour IOP data, ${ }^{11-13}$ however, this kind of monitoring is demanding and may be susceptible to bias.

Many studies have been aimed at finding some way of predicting the peak diurnal IOP. The water drinking test was considered to have an inadequate diagnostic value in the past $^{14}{ }^{15}$ but it has been proposed as a practical method to predict the IOP peak of the diurnal tension curve. Previous studies observed a correlation between the IOP peak in the diurnal tension curve and in the water drinking test. ${ }^{16}{ }^{17}$ This test may be used to access the efficacy of different forms of IOP reduction (clinical or surgical)..$^{17}$

To study the risk associated with diurnal IOP variations in patients with open angle glaucoma, we evaluated the water drinking test performance in open angle glaucoma eyes before a longitudinal follow up with periodic visual field examinations.

\section{METHODOLOGY}

One hundred and eighty patients with diagnosis of open angle glaucoma who were submitted to the water drinking test between January 1998 and December 2003 had their charts reviewed. Patients were selected based on inclusion and exclusion criteria. One eye of each patient was included in this study. If both eyes of the same patient were eligible, one was randomly selected.

All included eyes had a corrected visual acuity of 20/40 or better during the entire period of the study, spherical refraction within plus or minus 5.00 dioptres, and cylinder correction within plus or minus 3.00 dioptres. Eyes with closed or narrow angles at gonioscopy exam were excluded. Patients submitted to any surgical procedure or laser intervention during the evaluation period and patients with retinal disease, non-glaucomatous optic neuropathy, or any other disease that could interfere with visual field testing were also excluded.

Eyes with open angle glaucoma had to present an open angle at gonioscopy and a glaucomatous optic disk associated with reproducible glaucomatous visual field defect based on Anderson's criteria. ${ }^{19}$ Visual field defect was defined by the presence of a cluster of three or more non-edge points that have sensitivities with $\mathrm{p}<5 \%$ and one of the points has a sensitivity with $\mathrm{p}<1 \%$ or corrected pattern standard deviation (CPSD) value with $\mathrm{p}<5 \%$ or glaucoma hemifield test (GHT) outside normal limits.

All subjects were under clinical therapy and had IOP lower than $17 \mathrm{~mm} \mathrm{Hg}$ monitored by isolated measurements during the follow up period.

Abbreviations: CPSD, corrected pattern standard deviation; IOP, intraocular pressure. 
Table 1 Demographic data of open angle glaucoma eyes with and without visual field deterioration

\begin{tabular}{lccc}
\hline & $\begin{array}{c}\text { Eyes with visual field } \\
\text { deterioration }\end{array}$ & $\begin{array}{c}\text { Eyes without visual field } \\
\text { deterioration }\end{array}$ & p Value \\
\hline Number of eyes & $28(36.8 \%)$ & $48(63.2 \%)$ & \\
Age (years) (mean (SD)) & $66.8(13.7)$ & $67.4(10.7)$ & $0.83^{*}$ \\
Range (years) & $40-88$ & $39-86$ & \\
Sex (M/F) & $11 / 17$ & $30 / 18$ & $0.06 \dagger$ \\
Race & $25(89.3 \%)$ & $42(87.5 \%)$ & $0.82 \dagger$ \\
$\quad$ White & $3(10.7 \%)$ & $6(12.5 \%)$ & \\
$\quad$ Asians & $28.9(16.7)$ & $24.3(11.6)$ & $0.16^{*}$ \\
Period of follow up (months) (mean (SD)) & $4.7(2.2)$ & $0.37^{*}$ \\
Number of visual field exams (mean (SD)) & $4.3(1.8)$ & $-9.56(7.20)$ & $0.71^{*}$ \\
Initial MD (db) (mean (SD)) & $-8.97(5.63)$ & $6.67(4.50)$ & $0.37^{*}$ \\
Initial CPSD (db) (mean (SD)) & $7.73(5.53)$ & $12.6(2.0)$ & $0.57^{*}$ \\
Basal IOP (mmHg) (mean (SD)) & $12.9(2.7)$ & & \\
\hline${ }^{*}$ ANOVA. & & & \\
† $\chi^{2}$ test. & & & \\
MD, mean deviation; CPSD, correct pattern standard deviation; IOP, intraocular pressure; db, decibels.
\end{tabular}

Patients were submitted to the water drinking test at the beginning of the follow up period. The water drinking test was performed after obtaining a baseline IOP measurement. Patients had to drink one litre of tap water in 5 minutes. IOP was measured three times at 15 minutes intervals. Water drinking tests were performed between 4:00pm and 5:00pm. The maximum value of the three measurements was considered as the maximum IOP during the water drinking test.

IOP measurements were obtained with Goldmann applanation tonometry. Achromatic perimetry was performed with the Humphrey Visual Field Analyser (Humphrey Instruments, San Leandro, CA, USA) standard full threshold 24-2). All patients had previous experience with visual field tests and only eyes with reliable exams (less than $20 \%$ of fixation losses, and/or less than $33 \%$ of false positive and false negative rates) were included. The last reliable visual field exam performed before the evaluated period was considered as the baseline exam. The method adopted to evaluate visual field progression was based on Anderson's progression criteria $^{20}$ and was characterised by the rise of a new defect or worsening of a previous defect. A new defect was defined by the presence of a new cluster of three or more non-edge points that have sensitivities with $\mathrm{p}<5 \%$ with one of the points having a sensitivity with $\mathrm{p}<1 \%$. Worsening of a previously defective region was characterised by the deterioration of three or more points in that region by $10 \mathrm{db}$.

The end point for the eyes with visual field progression was considered the day of confirmation of the visual field deterioration. For the patients without visual field progression the last visual field exam performed during the evaluated period was considered the end point.

The number of eyes that developed visual field progression was determined. The performance of the water drinking test was compared between the eyes that developed visual field deterioration and those that did not.

Statistical analysis was performed with commercial software (SPSS 10.0, SPSS Inc, Chicago, IL, USA). Analysis of variance and $\chi^{2}$ test were used to compare, respectively, continuous and categorical demographic data between groups. The data included mean age, sex, ethnical group, basal IOP, number of antiglaucomatous drugs, initial mean deviation (MD) and correct pattern standard deviation (CPSD), period of follow up, and numbers of visual fields exams. Intraocular pressure peak and percentage of IOP fluctuation (intraocular pressure peak-baseline IOP/baseline IOP) during the water drinking test were evaluated in all eyes by analysis of covariance with initial IOP as a covariate.

\section{RESULTS}

Seventy six eyes of 76 patients were included in this study. Demographic data are presented in table 1. The mean age of all participants was 67.1 (SD 11.8) years. Thirty five (46.1\%) patients were women. The majority of subjects were White $(88.2 \%)$. The mean follow up period of all subjects was 26.0 (SD 13.8) months (5-67 months).

Twenty eight $(36.8 \%)$ eyes reached definite visual field progression based on the study criteria. No significant differences were found in the mean follow up time and the number of visual field exams performed between eyes with and without visual field deterioration (table 1). Similarly, no significant difference in the mean age, sex, race, basal IOP, initial MD, and CPSD was observed between groups (table 1).

From a overall baseline of $12.7 \mathrm{~mm} \mathrm{Hg}$, a mean (standard error of the mean) difference of 1.9 (SEM 0.6) $\mathrm{mm} \mathrm{Hg}$ in the mean IOP peak was observed between glaucomatous eyes that showed visual field progression and the ones that did not. This difference was significant $(\mathrm{p}=0.001$, ANCOVA; $95 \%$ CI 0.8 to 3.0 ) (see table 2 ).

In the same way, a difference of $16.8 \%$ (SEM $4.6 \%$ ) in the mean percentage of IOP variation was observed between glaucomatous eyes which showed visual field deterioration and the ones that did not progress. This difference was also considered significant $(\mathrm{p}<0.001$, ANCOVA; $95 \%$ CI 7.7 to 26.0).

The mean (standard deviation) number of antiglaucomatous drugs used in the eyes that demonstrated visual field progression was 2.4 (SD 1.4) compared with 2.1 (SD 1.3) in the non-progressive group $(\mathrm{p}=0.42)$. Prostaglandins were part of the clinical treatment in $15(53.6 \%)$ of the 28 eyes that showed visual field progression and in $32(66.7 \%)$ of the 48 eyes that remained stable $(p=0.26)$. No significant difference was observed in the number of eyes using beta blockers, carbonic anhydrase inhibitors, and alpha agonists between the two groups (table 3 ). The number of eyes submitted to

Table 2 Water drinking test results: IOP peaks and percentage of IOP fluctuations

\begin{tabular}{|c|c|c|c|}
\hline Water drinking test & $\begin{array}{l}\text { With VF } \\
\text { deterioration }\end{array}$ & $\begin{array}{l}\text { Without VF } \\
\text { deterioration }\end{array}$ & $\begin{array}{l}\text { p Value at } \\
95 \% \mathrm{Cl}\end{array}$ \\
\hline $\begin{array}{l}\text { IOP peak mm Hg (mean } \\
\text { (SEM)) }\end{array}$ & $16.8(0.4)$ & $14.9(0.3)$ & $<0.01^{*}$ \\
\hline $\begin{array}{l}\text { \% IOP fluctuation } \mathrm{mm} \mathrm{Hg} \\
\text { (mean (SEM)) }\end{array}$ & $34.0(3.6)$ & $17.2(2.8)$ & $<0.01^{*}$ \\
\hline
\end{tabular}


Table 3 Percentage of eyes under each major kind of topical antiglaucoma drugs

\begin{tabular}{llll}
\hline Topical antiglaucoma drugs & $\begin{array}{l}\text { With VF } \\
\text { deterioration }\end{array}$ & $\begin{array}{l}\text { Without VF } \\
\text { deterioration }\end{array}$ & $\begin{array}{l}\mathbf{p} \text { Value at } \\
\mathbf{9} \text { Cl }\end{array}$ \\
\hline Beta blockers & $75.0 \%$ & $64.6 \%$ & 0.35 \\
Carbonic anhydrase inhibitors & $67.8 \%$ & $45.8 \%$ & 0.06 \\
Alpha agonists & $28.6 \%$ & $16.7 \%$ & 0.2 \\
\hline$\chi^{2}$ test. & & & \\
VF, visual field; $\mathrm{Cl}$, confidence interval. & & \\
\hline
\end{tabular}

glaucoma surgery (trabeculectomy) before the studied period was seven (25\%) in the progressive group and $12(25 \%)$ in the non-progressive group $(\mathrm{p}=0.84)$.

Among eyes that reached definite visual field progression, $25 \%(7 / 28)$ showed IOP $>21 \mathrm{~mm} \mathrm{Hg}$ during the water drinking test compared with $4.2 \%(2 / 48)$ observed in the group that remained stable $(\mathrm{p}=0.007)$. In this group, four of $48(8.3 \%)$ eyes had an IOP variation $\geqslant 6 \mathrm{~mm} \mathrm{Hg}$ in the water drinking test compared with $35.7 \%(10 / 28)$ of eyes that demonstrated visual field progression $(\mathrm{p}=0.003)$.

\section{DISCUSSION}

The water drinking test is a provocative test that was widely used a few decades ago to help in the diagnosis of open angle glaucoma, ${ }^{15}{ }^{16}$ but was found to be inadequate due to many false positive and false negative results in 10 year prospective studies. ${ }^{15}$ However, after some years, the emphasis on the value of this test has changed. As a result of the correlation with the diurnal tensional curve, ${ }^{16}$ the water drinking test has been proposed as an alternative method to check IOP control.

In this study we performed the water drinking test in glaucomatous eyes with an IOP lower than $17 \mathrm{~mm} \mathrm{Hg}$ under clinical and/or surgical therapy. Our findings revealed a significant difference in the results of the water drinking test between eyes that showed glaucoma progression compared with eyes that remained stable.

Previous studies had already suggested the importance of the water drinking test to determine a risk factor for the development of glaucomatous visual field defect. In the 1980s, based on a large prospective study (Collaborative Glaucoma Study), Armaly and coworkers ${ }^{21} 22$ reported the pressure change after drinking water as one of five potential risk factors significantly related to the development of glaucomatous visual field defects in patients with ocular hypertension.

Another study performed by Yoshikawa et al ${ }^{23}$ in normal tension glaucoma patients showed that the maximum IOP levels after the water drinking test in patients with progressive visual field loss was significantly greater than the levels observed for the non-progressive group. Unlike their study, we also included open angle glaucoma patients with an IOP higher than $21 \mathrm{~mm} \mathrm{Hg}$ at the time of diagnosis. In agreement with their results, we observed a difference of almost $2 \mathrm{~mm} \mathrm{Hg}$ in the IOP peak during the water drinking test between eyes that progressed and the ones that did not. In addition, more eyes with IOP peaks higher than $21 \mathrm{~mm} \mathrm{Hg}$ during the water drinking test were observed in the progressive group.

The importance of IOP peaks in the development of glaucoma progression has been already reported by Zeimer et $a l^{11}$ and also by Martinez-Belló et al. ${ }^{24}$ In our study, we did not observe a difference in the mean basal IOP obtained during isolated office visits between the two groups, but our results revealed that the percentage of IOP variation during the water drinking test in eyes with visual field progression was almost twice the value observed in the other group.
Despite the results obtained by our study and previous reports, the mechanisms involved in the water drinking test are not completely understood. After drinking water or any hypotonic fluid, there is absorption of water into blood and body tissues including the eye. This is associated with a consequent rise in IOP. The ability of the eye to recover from this transient IOP rise depends on the outflow facility. The rapid inflow of aqueous humour and the low facility of outflow in the glaucomatous eye may lead, at least in part, to larger IOP fluctuation.

Recently, Brubaker ${ }^{25}$ proposed that the water drinking test could be used as an indirect measurement of outflow facility to compare the IOP responses of glaucoma eyes to different drugs. Drugs such as prostaglandins improve the outflow facility and are expected to show less IOP variation secondary to water challenge. Although eyes were under different hypotensive medication regimen in this study, no significant difference in the frequency of prostaglandins as part of the clinical treatment was observed between the two groups. The presence or not of any filtration surgery should also be considered when comparing water drinking test results between eyes. Earlier studies already showed a relatively small range of diurnal IOP variation in trabeculectomised eyes. ${ }^{17}{ }^{26}$ In our study no significant difference in the number of eyes submitted to glaucoma surgery (trabeculectomy) before the studied period was found between the progressive group and the non-progressive group, reducing possible bias.

This study may be vulnerable to some other biases related to a retrospective analysis. Although no significant difference was found in the number of visual fields performed between eyes that progressed and the ones that remained stable, visual field examination was not performed at the same intervals in all patients. Despite this, no significant difference between groups was found in other baseline variables that could possibly influence the outcome parameters of the water drinking test.

In this study we did not try identify which value of IOP peak or IOP variation would suggest a higher risk of progression. Cutoffs may not be useful when evaluating water drinking test results to assess the risk of progression for all individuals. Each result of the water drinking test may provide important information when evaluating treatment efficacy for a specific patient once the clinician has established the target pressure.

In summary, the water drinking test is a low cost and feasible test and may reflect one of the important and common disturbances to steady state IOP during a common activity of daily living: drinking water. ${ }^{25}$ Our findings suggest that the emphasis on the value of the water drinking test should be changed. It may be useful as a complementary exam to check IOP control in the clinical practice. The peak and percentage of IOP variation of this test should be considered when analysing risk factors for glaucoma progression. Future randomised controlled longitudinal studies may give a better evaluation of this possibility.

\section{Authors' affiliations}

R SusannaJr, R M Vessani, L Sakata, L C Zacarias, M Hatanaka, Glaucoma Service, Ophthalmology Department, University of São Paulo, Brazil

\section{REFERENCES}

1 Liu JH. Diurnal measurement of intraocular pressure. J Glaucoma $2001 ; 10:$ S39-41

2 The Advanced Glaucoma Intervention Study (AGIS): 7. The relationship between control of intraocular pressure and visual field deterioration. The AGIS Investigators. Am J Ophthalmol 2000;130:429-40.

3 The effectiveness of intraocular pressure reduction in the treatment of normaltension glaucoma. Collaborative Normal-Tension Glaucoma Study Group. Am J Ophthalmol 1998;126:498-505. 
4 Leske MC, Heiil A, Hussein M, et al. Factors for glaucoma progression and the effect of treatment: the early manifest glaucoma trial. Arch Ophthalmol 2003; 121:48-56.

5 Kass MA, Kolker AE, Becker B. Prognostic factors in glaucomatous visual field loss. Arch Ophthalmol 1976;94:1274-6.

6 Werner EB, Drance SM. Progression of glaucomatous field defects despite successful filtration. Can J Ophthalmol 1977;12:275-80.

7 Chauhan BC, Drance SM. The relationship between intraocular pressure and visual field progression in glaucoma. Graefes Arch Clin Exp Ophthalmol 1992;230:521-6.

8 Brubaker RF. Delayed functional loss in glaucoma. LII Edward Jackson Memorial Lecture. Am J Ophthalmol, 1996;121:473-83.

9 Zeimer R. Circadian variations in intraocular pressure. In: Ritch R, Shields MB Krupin T, eds. The glaucomas. St Louis: CV Mosby Co, 1996.

10 Hughes E, Spry P, Diamond J. 24-hour monitoring of intraocular pressure in glaucoma management: a retrospective review. J Glaucoma 2003;12:232-6.

11 Zeimer RC, Wilensky JT, Gieser DK, et al. Association between intraocular pressure peaks and progression of visual field loss. Ophthalmology 1991;98:64-9.

12 Asrani S, Zeimer R, Wilensky J, et al. Large diurnal fluctuations in intraocular pressure are an independent risk factor in patients with glaucoma. J Glaucoma 2000;9:134-42.

13 Zeimer RC, Wilensky JT, Gieser DK, et al. Application of a self-tonometer to home tonometry. Arch Ophthalmol 1986;104:49-53.

14 Rasmussen KE, Jorgensen HA. Diagnostic value of the water drinking test in early detection of simple glaucoma. Acta Ophthalmol (Copenh) 1976;54:160-6.
15 Roth JA. Inadequate diagnostic value of the water-drinking test. Br J Ophthalmol 1974;58:55-61.

16 Miller $\mathbf{D}$. The relationship between diurnal tension variation and the waterdrinking test. Am J Ophthalmol 1964;58:243-6.

17 Medeiros FA, Pinheiro A, Moura FC, et al. Intraocular pressure fluctuations in medical versus surgically treated glaucomatous patients. J Ocul Pharmacol Ther 2002;18:489-98.

18 Frankelson EN. The role of the water test in evaluation of glaucoma control Can J Ophthalmol 1974;9:408-10.

19 Anderson DR. Automated Static Perimetry. St Louis: Mosby-Year Book, 1992.

20 Anderson DR. Automated Static Perimetry, Second Edition. St Louis: MosbyYear Book, 1999.

21 Armaly MF. Lessons to be learned from the Collaborative Glaucoma Study. Surv Ophthalmol 1980;25:139-44.

22 Armaly MF, Krueger DE, Maunder L, et al. Biostatistical analysis of the collaborative glaucoma study. I. Summary report of the risk factors for collaborative glaucoma study. I. Summary report of the risk factors for
glaucomatous visual-field defects. Arch Ophthalmol 1980;98:2163-71.

23 Yoshikawa K, Inove T, Inove Y. Normal tension glaucoma: the value of predictive tests. Acta Ophthalmol (Copenh) 1993;71:463-70.

24 Martinez-Bello C, Chauhan BC, Nicolela MT, et al. Intraocular pressure and progression of glaucomatous visual field loss. Am J Ophthalmol 2000;129:302-8.

25 Brubaker RF. Importance of outflow facility. IGR 2001;3:1.

26 Saiz A, Alcuaz A, Maquet JA, et al. Pressure-curve variations after trabeculectomy for chronic primary open-angle glaucoma. Ophthalmic Surg 1990;21:799-801. 